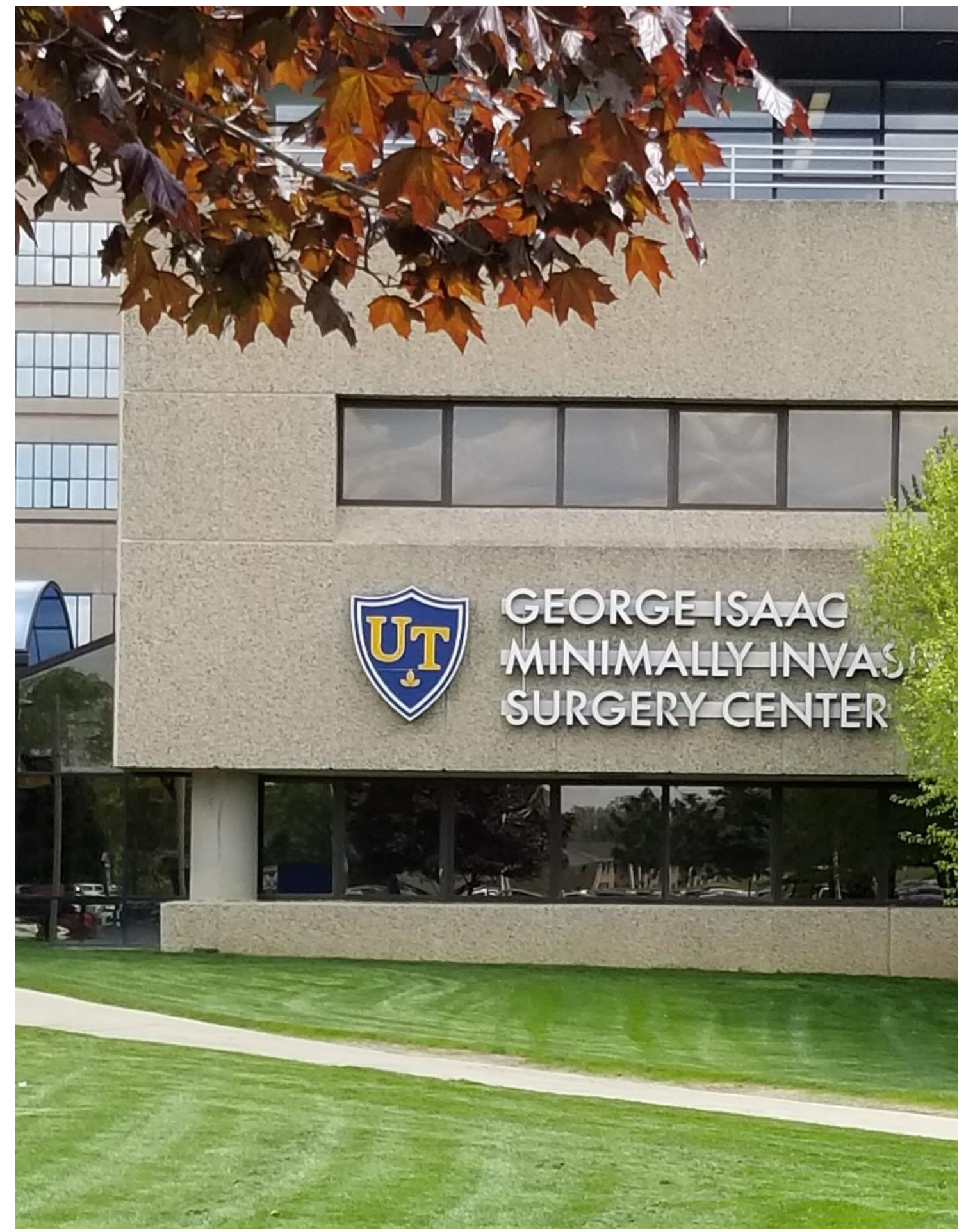




\title{
Streptococcus pneumonia urinary tract infection in 3-year-old girl
}

\author{
Daniel Lubarsky, ${ }^{a},{ }^{1}$ Daniel Garcia, ${ }^{b}$ and Deepa Mukundan $c$
}

${ }^{a}$ M.D. Candidate, Class of 2021, The University of Toledo Heath Science Campus, Toledo, OH, USA, ${ }^{b}$ Department of Pediatrics, University of Toledo Health Science Campus, Toledo, $\mathrm{OH}, \mathrm{USA}$, and ${ }^{c}$ Department of Pediatric Infectious Disease, The University of Toledo Heath Science Campus, Toledo, OH, USA

\begin{abstract}
Streptococcus pneumonia is a gram-positive bacterium most commonly associated with respiratory tract infections such as acute otitis media, sinusitis, and pneumonia. While a common cause of upper respiratory infections, the current Infectious Diseases Society of America (IDSA) guidelines on urinary tract infections (UTIs) do not cite Streptococcus pneumoniae as a cause for UTIs in children. In addition, previous research associating Streptococcus pneumonia with UTIs is scarce. We report the case of a three-year-old girl presenting to the emergency room with abdominal pain, vomiting, and fever. Her clinical picture was consistent with right-sided pyelonephritis. A urine analysis (UA) was conducted which showed trace leukocyte esterase, $150 \mathrm{mg} / \mathrm{dL}$ ketones, 21-50 WBCs, and negative nitrites. A blood culture returned positive for streptococcus pneumoniae along with right kidney hydronephrosis on ultrasound. This patient presented with a full clinical picture of pyelonephritis, fever, leukocytosis, elevated C-reactive protein (CRP) and procalcitonin levels. Although the urine culture did not grow any definitive single bacterium as a causative agent, due to the presentation of pyelonephritis along with CVA tenderness, dysuria, and a positive blood culture, we conclude the patient was exhibiting urosepsis due to streptococcus pneumoniae. Upon discovering previous case reports noting $\mathrm{S}$. pneumoniae as a possible agent for UTIs, we believed this bacterium is contributing to the patient's symptoms. While streptococcus pneumoniae is rarely reported as a cause of UTIs, we believe there is sufficient evidence that demonstrates streptococcus pneumoniae as a possible contributing cause of UTIs, especially if the patient has a coinciding septic picture.
\end{abstract}

\section{Streptococcus pneumonia | Urinary Tract Infection |}

$\mathbf{S}$ treptococcus pneumonia is a gram positive bacterium most commonly associated with respiratory tract infections such as acute otitis media, sinusitis, and pneumonia (1). While a common cause of upper respiratory infections, the current Infectious Diseases Society of America (IDSA) guidelines on urinary tract infections do not cite Streptococcus pneumoniae as a cause for UTIs in children (2). The main bacterial agents associated with uncomplicated UTIs in children are Escherichia coli, Klebsiella pneumoniae, and Enterobacter species (3). According to guidelines released by the American Academy of Pediatrics (AAP), a UTI is defined as bacteriuria $\left(>10^{5} \mathrm{CFU} / \mathrm{mL}\right)$ of one uropathogen along with dysuria, pyuria, and urgency (4). Fever, urinary symptoms, flank pain and an abnormal urinalysis would appropriately raise suspicion for pyelonephritis (5).

Previous research associating Streptococcus pneumonia with UTIs has been scarce. Miller et al. published a report on pneumococcosuria in children. After examining 53,499 urine samples from children, $43(0.08 \%)$ positive for Streptococcus pnuemoniae, the report concluded that Streptococcus pneumoniae was more likely to be a contaminant from perineal colonization (6). Nguyen and Penn published a paper on pneumococcosuria in adults. The results were obtained from 22,744 urine samples obtained over four years showing the frequency of Streptococcus pneumoniae to be $38(0.18 \%)$ of 22,744 samples (7). Dufke et al. described an 82 year old male with pyelonephritis and urosepsis found to have been caused by serotype 6A of Streptococcus pneumoniae (8). In 2011, Burckhardt and Zimmerman published a report on 3 children with history of chronic kidney disease who showed high numbers of Streptococcus pneumoniae in their urine (9). The following year Krishna et al. reported on 6 more cases of pneumococusuria with the isolation of Streptococcus pnuemoniae in their urine samples (10). Finally, in 2016 Burkhardt et al. conducted an analysis on 110,000 urine specimens and identified 26 urine samples $(0.02 \%)$ contain Streptococcus pneumoniae (11).

\section{Case report}

Patient information. Age: 3 years old. Gender: Female. Ethnicity: Caucasian. Related Medical Problems: None.

Objective. Discuss differential of UTI in pediatric patient and Streptococcus pneumonia as a possible contributing cause of UTIs

Case. A three-year-old female with no significant past medical history presented to the emergency room with abdominal pain, emesis, and fever. One week prior to admission she visited a lake with her family, and one day prior the patient was at a family baby shower playing with other children of no known illnesses. Later that night the patient became fatigued, followed by emesis and complaints of abdominal pain. Due to the persistent abdominal pain and emesis, she was taken to an outside emergency room. In the emergency room, a complete blood count (CBC) showed $41.910^{3}$ WBCs ( $88 \%$ neutrophils) and $50510^{3}$ platelets with all other values within normal limits. A comprehensive metabolic panel displayed a creatinine of $0.39 \mathrm{mg} / \mathrm{dL}$, and an elevated alkaline phosphate of 297 units/L with other values within normal limits. A urine analysis (UA) was conducted which showed trace leukocyte esterase, 150 $\mathrm{mg} / \mathrm{dL}$ ketones, 21-50 WBCs, and negative nitrites. Additionally, the patient's CRP level was $9.81 \mathrm{mg} / \mathrm{dL}$ (reference range 0.00-1.00) A bagged urine specimen and blood cultures were also obtained at the time. The patient was given one dose of Ceftriaxone $75 \mathrm{mg} / \mathrm{kg}$ intravenously for a presumed urinary tract infection and was admit-

All authors contributed to this paper. ${ }^{1}$ To whom correspondence should be sent: Daniel.Lubarsky@rockets.utoledo.edu

The authors declare no conflict of interest. Submitted: 07/27/2019, published: 10/22/2019.

Freely available online through the UTJMS open access option 
ted to the local children's hospital for further management.

Upon further questioning the patient was additionally positive for subjective fever, right ear pain, abdominal pain, vomiting, and back pain along with dysuria. On exam the patient's abdomen was tender to palpation, primarily in the suprapubic and right costovertebral angle (CVA) areas. Tympanic membranes appeared normal and the rest of the physical exam was unremarkable. The patient's repeat CBC showed significant leukocytosis of $16.110^{9} / \mathrm{L}(81.8 \%$ neutrophils), platelet count of $35710^{9} / \mathrm{L}$, hemoglobin count of 10.9 $\mathrm{g} / \mathrm{dL}$, hematocrit of $32.4 \%$, an elevated CRP of $6.7 \mathrm{mg} / \mathrm{dL}$ (reference range $0.000-0.744 \mathrm{mg} / \mathrm{dL}$ ) as well as an elevated procalcitonin of $10.29 \mathrm{ng} / \mathrm{mL}$ (reference range $<.05 \mathrm{ng} / \mathrm{mL}$ ). A bagged urine culture specimen from the outside emergency room demonstrated $>80,000$ colony forming units of mixed flora suggesting contamination and the blood culture returned positive for Streptococcus pneumoniae. Based on clinical findings of fever, abdominal pain, vomiting and CVA tenderness, a complete retroperitoneal ultrasound was done to examine for pyelonephritis. The ultrasound showed trace fluid in the right renal pelvis without obstruction as well as right sided hydronephrosis (shown in Figure 1) with no other abnormalities. After staying afebrile for a 36-hour period, the patient was prescribed a 10-day course of Amoxicillin for urosepsis due to Streptococcus pneumoniae and discharged.

\section{Discussion}

This patient presented with a full clinical picture of pyelonephritis, fever, leukocytosis, elevated CRP and procalcitonin levels, and later found to be Streptococcus pneumoniae positive. Although the urine culture did not grow any definitive single bacterium as a causative agent, due to the presentation of pyelonephritis with CVA tenderness, dysuria, and a positive blood culture, we conclude the patient was exhibiting urosepsis due to Streptococcus pneumoniae. Upon discovering previous case reports noting Streptococcus pneumoniae as a possible agent for UTIs, we believed this bacterium to be contributing to the patient's symptoms. Though Streptococcus pneumoniae has only been a rarely reported cause of UTIs, we believe there is sufficient evidence that demonstrates Streptococcus pneumoniae to at least be considered a possible contributing cause of UTIs, especially if the patient has a coinciding septic picture.

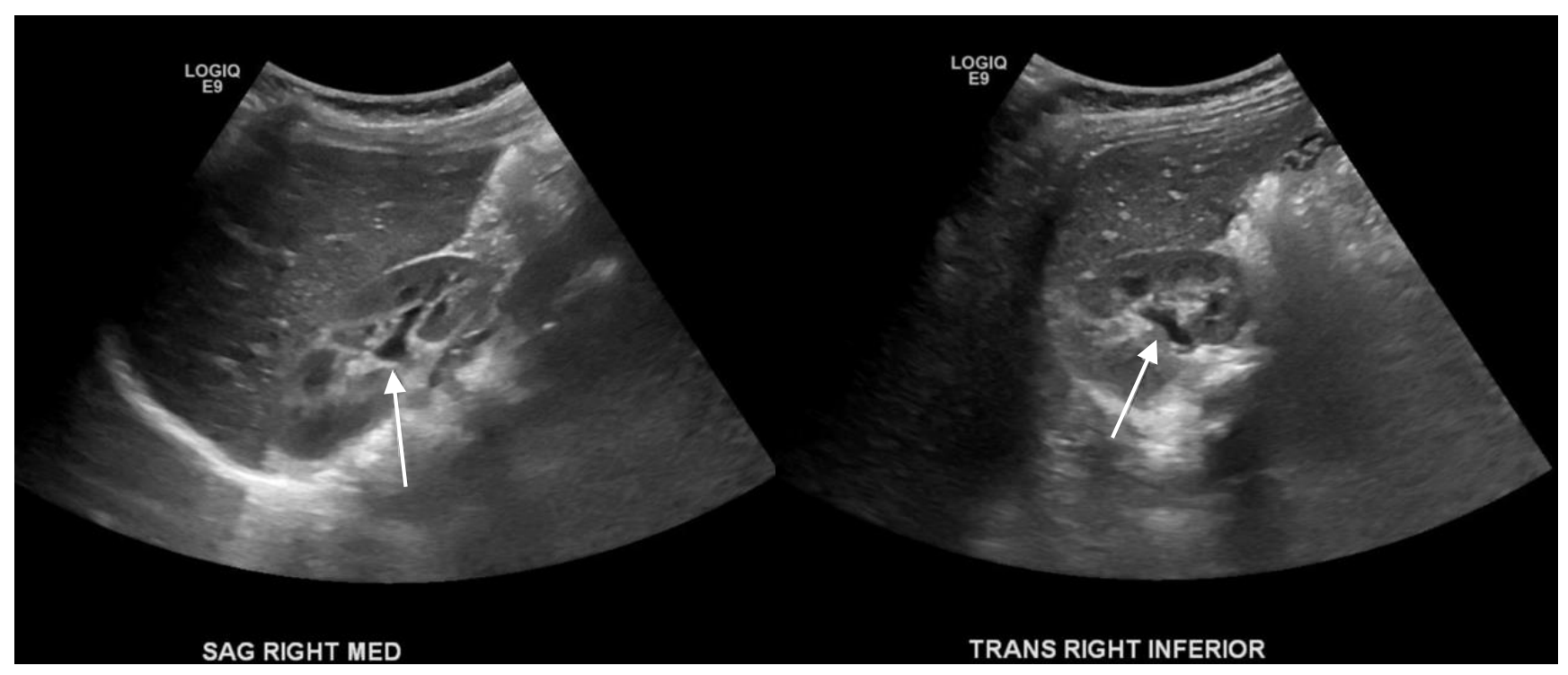

Figure 1. Ultrasound of right kidney demonstrating hydronephrosis (white arrows).

\section{Conclusion}

In summation, our goal is to present Streptococcus pneumoniae as a potential rare cause of pediatric UTIs. In our case, we concluded our patient presented with a full clinical picture of pyelonephritis, fever, leukocytosis, elevated CRP and procalcitonin level, most likely caused due to Streptococcus pneumoniae. Physicians should be aware of this bacterium as contributing cause for UTIs and consider it in their differential in pediatric patients.

\section{Conflict of interest}

Authors declare no conflict of interest.

\section{Authors' contributions}

DL wrote the manuscript, DG and DM revised the manuscript. All authors read and approved the final document. 
1. Maraqa NF (2014) Pneumococcal infections. Pediatr Rev 35, 299-310.

2. Gupta $K$ et al. (2011) International clinical practice guidelines for the treatment of acute uncomplicated cystitis and pyelonephritis in women: A 2010 update by the Infectious Diseases Society of America and the European Society for Microbiology and Infectious Diseases. Clin Infect Dis 52, e103-120.

3. Robinson JL et al., (2014) Urinary tract infections in infants and children: Diagnosis and management. Paediatr Child Health 19, 315-325.

4. Robert KB, (2012) Revised AAP Guideline on UTI in Febrile Infants and Young Children. Am Fam Physician 86, 940-946.

5. Chishti AS, Maul EC, Nazario RJ, Bennett JS, Kiessling SG (2010) A guideline for the inpatient care of children with pyelonephritis. Ann Saudi Med 30, 341-349.

6. Miller MA, Kaplan BS, Sorger S, Knowles KF (1989) Pneumococcosuria in children. J Clin Microbiol 27, 99-101.
7. Nguyen VQ, Penn RL (1988) Pneumococcosuria in adults. J Clin Microbiol 26, 1085-1087.

8. Dufke S, Kunze-Kronawitter $\mathrm{H}$, Schubert S (2004) Pyelonephritis and urosepsis caused by Streptococcus pneumoniae. J Clin Microbiol 42, 4383-4385.

9. Burckhardt I, Zimmermann S (2011) Streptococcus pneumoniae in urinary tracts of children with chronic kidney disease. Emerg Infect Dis 17, 120-122.

10. Krishna $S$ et al., (2012) Pneumococcusuria: From bench to bedside. Indian $J$ Med Microbiol 30, 96-98.

11. Burckhardt I, Panitz J, van der Linden M, Zimmermann S (2016) Streptococcus pneumoniae as an agent of urinary tract infections - a laboratory experience from 2010 to 2014 and further characterization of strains. Diagn Microbiol Infect Dis 86, 97-101. 